\title{
Edge Physics Studies on the NSTX Spherical Tokamak
}

In this funding period, activities were focused on processing data for publication, writing code to facilitate processing of data in the future as programmatic needs arise, and closing down grant activities.

\section{Summary of Results and Highlights}

- Comparing plasma profile and fluctuation data to turbulence simulation codes (SOLT) was carried out ${ }^{1}$. SOLT is an electrostatic two-dimensional fluid turbulence model that contains curvature-driven-interchange modes, sheath losses, and both perpendicular turbulent diffusive and convective (blob) transport. Midplane SOL profiles of density, temperature, and parallel heat flux are obtained from the simulation and compared with experimental results to study the scaling of the heat-flux width with power and plasma current. It is concluded that midplane turbulence is the main contributor to the SOL heat-flux width for the low power H-mode discharges studied, while additional physics is required to fully explain the plasma current scaling of the SOL heat-flux width observed experimentally in higher power discharges. Intermittent separatrix-spanning convective cells are found to be the main mechanism that sets the near-SOL width in the simulations.

- The probe system built for NSTX was described in Review of Scientific Instruments ${ }^{2}$. We describe a fast reciprocating Langmuir probe and drive system, which has four main new features: (1) use of high-temperature, vacuum, circuit boards instead of cables to reduce weight and increase to 21 the number of possible connections, (2) rotatable and removable shaft, (3) 10 tip construction with designed hardware bandwidth up to $10 \mathrm{MHz}$, and (4) a detachable and modular tip assembly for easy maintenance. The probe is mounted in a fast pneumatic drive capable of speeds $\sim 7 \mathrm{~m} / \mathrm{s}$ and $\sim 20 \mathrm{~g}$ 's acceleration in order to reach the scrape-off layer (SOL) and pedestal regions and remain inserted long enough to obtain good statistics while minimizing the heat deposition to the tips and head in a power density environment of 1-10 MW/ $/ \mathrm{m}^{2}$. The National Spherical Torus Experiment SOL features electron temperature, $T_{e} \sim 10-30 \mathrm{eV}$, and electron density, $n_{e} \sim 0.1-5 \times 10^{12}$ $\mathrm{cm}^{-3}$ while the pedestal features $n_{e} \sim 0.5-1.5 \times 10^{13} \mathrm{~cm}^{-3}$ and $T_{e} \sim 30-150 \mathrm{eV}$. The probe described here has ten tips which obtain a wide spectrum of plasma parameters: electron temperature profile $T_{e}(r)$, electron density profile $n_{e}(r)$ and Mach number profile $M(r)$, floating potential $V_{f}(r)$, poloidal and radial electric field profiles $E_{\theta}(r)$ and $E_{\rho}(r)$, saturation current profile $I_{\text {sat }}(r)$, and their fluctuations up to $3 \mathrm{MHz}$. We describe the probe and show representative radial profiles of various parameters.

- The dependence of various SOL widths on the line-averaged density (ne) and plasma current $\left(I_{\mathrm{p}}\right)$ for the quiescent H-mode plasmas with Type-V ELMs in 
the National Spherical Torus Experiment (NSTX) was investigated ${ }^{3}$. The heat flux SOL width $\left(\lambda_{q}\right)$, measured by the IR camera, is virtually insensitive to ne and has a strong negative dependence on $I_{\mathrm{p}}$. This insensitivity of $\lambda_{\mathrm{q}}$ to ne is consistent with the scaling law from JET H-mode plasmas that shows a very weak dependence on the upstream density. The electron temperature, ion saturation current density, electron density, and electron pressure decay lengths ( $\lambda_{\mathrm{Te}}, \lambda_{\text {jsat }}, \lambda_{\text {ne, }}$ and $\lambda_{\text {pe, }}$ respectively) measured by the probe showed that $\lambda_{\mathrm{Te}}$ and $\lambda_{\text {jsat }}$ have strong negative dependence on $I_{\mathrm{p}}$, whereas $\lambda_{\text {ne }}$ and $\lambda_{\mathrm{pe}}$ revealed only a little or no dependence. The dependence of $\lambda_{\mathrm{Te}}$ on $I_{\mathrm{p}}$ is consistent with the scaling law in the literature.

- A review on turbulence across devices such as NSTX, DIII-D, ALCATOR-C MOD, etc supports the mechanisms of intermittency as a universal transport feature produce by interchange instability and controlling many the SOL characteristics ${ }^{4}$.

- The physics of parallel heat transport was tested in the scrape-off layer (SOL) plasma of NSTX by comparing the upstream electron temperature $\left(T_{e}\right)$ and density $\left(n_{e}\right)$ profiles measured by the midplane reciprocating probe to the heat flux $\left(q_{\perp}\right)$ profile at the divertor plate measured by an infrared camera ${ }^{5}$. It is found that electron conduction explains the near SOL width data reasonably well while the far SOL, which is in the sheath limited regime, requires an ion heat flux profile broader than the electron one to be consistent with the experimental data. The measured plasma parameters indicate that the SOL energy transport should be in the conduction-limited regime for $R-R_{\text {sep }}$ (radial distance from the separatrix location) $<2-3 \mathrm{~cm}$. The SOL energy transport should transition to the sheath-limited regime for $R-R_{\text {sep }}>2-3 \mathrm{~cm}$. The $T_{e}, n_{e}$, and $q_{\perp}$ profiles are better described by an offset exponential function instead of a simple exponential. The conventional relation between midplane electron temperature decay length $\left(\lambda_{T e}\right)$ and target heat flux decay length $\left(\lambda_{q}\right)$ is $\lambda_{T e}=7 / 2 \lambda_{q}$, whereas the newly derived relation, assuming offset exponential functional forms, implies $\lambda_{T e}=(2-$ 2.5) $\lambda_{q}$. The measured values of $\lambda_{T e} / \lambda_{q}$ differ from the new prediction by $25 \%-$ $30 \%$. The measured $\lambda_{q}$ values in the far SOL $\left(R-R_{\text {sep }}>2-3 \mathrm{~cm}\right)$ are $9-10 \mathrm{~cm}$, while the expected values are $2.7<\lambda_{q}<4.9 \mathrm{~cm}$ (for the sheath-limited regime). We propose that the ion heat flux profile is substantially broader than the electron heat flux profile as an explanation for this discrepancy in the far SOL.

- Detailed profile data from probe measurements was used to evaluate gas puff imaging models ${ }^{6}$. Gas puff imaging (GPI) experiments are designed to provide high time and space resolution data on the structure of plasma turbulence in the plane perpendicular to the magnetic field. We first examine the temporal behavior of the helium atoms used as the emitting species for GPI and show that for the time scales of interest ( $\gtrsim 1 \mu \mathrm{s})$, the atomic physics model underlying the conventional interpretation of GPI is valid. Second, we continue the Monte Carlo neutral transport simulations of the GPI diagnostic 
begun in [D.P. Stotler et al., Contrib. Plasma Phys. 44 (2004) 294]. The radial characteristics of the simulated emission clouds match observations to within the estimated errors. The upshot of these two results is that the technique for unfolding the 2-D, time-dependent plasma density and temperature data from helium GPI emission, relying on this atomic physics model and utilizing the simulated neutral density data from DEGAS 2 , is valid.

\section{Other Activities}

The UCSD hardware, repciprocating probe, control system and electronics was removed from NSTX in preparation for the upgrade.

\section{References:}

${ }^{1}$ Reduced model simulations of the scrape-off-layer heat-flux width and comparison with experiment Myra, J.R.; Russell, D.A.; D’Ippolito, D.A.; et al., Physics of Plasmas, Volume: 18 Issue 1, pp. 012305, 2011.

${ }^{2}$ Fast scanning probe for the NSTX spherical tokamak Boedo, J.A.; Crocker, N.; Chousal, L.; et al., Review of Scientific Instruments Volume: 80 Issue: 12 pp 1235062009.

3 Dependence of SOL widths on plasma current and density in NSTX H-mode plasmas Ahn, J.-W.; Maingi, R.; Boedo, J.A.; et al., Journal of Nuclear Materials Volume: 390-391 pp: 421, 2009.

${ }^{4}$ Edge turbulence and SOL transport in tokamaks Boedo, J.A., Journal of Nuclear Materials, Volume: 390-391, pp: 29-37, 2009

${ }^{5}$ The role of parallel heat transport in the relation between upstream scrape-off layer widths and target heat flux width in H-mode plasmas of the National Spherical Torus Experiment , Ahn, J.-W.; Boedo, J.A.; Maingi, R.; et al., Physics of Plasmas, Volume: 15, Issue: 12, pp 122507, 2008

${ }^{6}$ Progress towards the validation of models of the behavior of neutral helium in gas puff imaging experiments Stotler, D.P.; Boedo, J.; LeBlanc, B.; et al., Journal of Nuclear Materials, Volume: 363-365, pp: 686-92, 2007. 